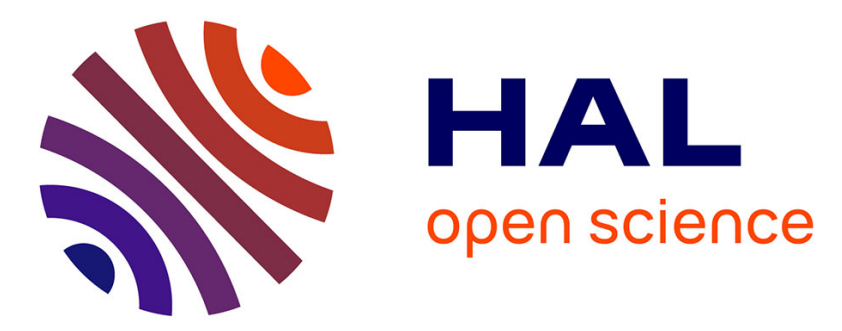

\title{
Transparency in Public Life. A Quantum Cognition Perspective
}

Ariane Lambert-Mogiliansky, François Dubois

\section{To cite this version:}

Ariane Lambert-Mogiliansky, François Dubois. Transparency in Public Life. A Quantum Cognition Perspective. 2015. halshs-01064980v2

\section{HAL Id: halshs-01064980 \\ https://shs.hal.science/halshs-01064980v2}

Preprint submitted on 11 Mar 2015

HAL is a multi-disciplinary open access archive for the deposit and dissemination of scientific research documents, whether they are published or not. The documents may come from teaching and research institutions in France or abroad, or from public or private research centers.
L'archive ouverte pluridisciplinaire HAL, est destinée au dépôt et à la diffusion de documents scientifiques de niveau recherche, publiés ou non, émanant des établissements d'enseignement et de recherche français ou étrangers, des laboratoires publics ou privés. 


\title{
PARISSCHOOL OF ECONOMICS
}

WORKING PAPER Nº $2014-29$

Transparency in Public Life A Quantum Cognition Perspective

\author{
Ariane Lambert-Mogiliansky \\ François Dubois
}

JEL Codes:

Keywords: learning, quantum cognition, transparency

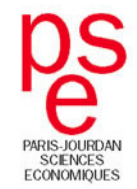




\title{
Transparency in Public Life A Quantum Cognition Perspective
}

\author{
Ariane Lambert-Mogiliansky ${ }^{1}$ and François Dubois ${ }^{2,3}$ \\ 1 Paris School of Economics, \\ 48 Bd Jourdan, 75014 Paris, France. \\ 2 Department of Mathematics, University Paris Sud, \\ Bât. 425, F-91405 Orsay Cedex, France. \\ ${ }^{3}$ Conservatoire National des Arts et Métiers, Paris, France, \\ Structural Mechanics and Coupled Systems Laboratory. \\ alambert@pse.ens.fr, francois.dubois@math.u-psud.fr.
}

29 August $2014^{1}$

\begin{abstract}
In this paper we investigate the implications of assuming that citizens are cognitively constrained for transparency in public life. We model cognitive limitations as reflecting a quantum property of people's mental representations of the world. There exists a multiplicity of incompatible (Bohr) complementary mental representations of a situation. As a consequence the framing of information plays a crucial role. We show that additional information can be detrimental to a quantum cognitively constrained agent: he may become more confused. We suggest some implications for the design of a public agency's website.
\end{abstract}

Keywords: learning, quantum cognition, transparency.

AMS classification: 81Q99, 91C99.

\section{Introduction}

Transparency is among the most debated issues in modern public life. The development of information technology has given rise to hopes for a modern truly democratic state based on transparency. As noted by Beth Noveck [18], real applications have fallen short for those hopes. In part we suggest that this may be due to a narrow understanding of the concept of transparency. This paper is a contribution to the exploration of the links between access to information and transparency. The starting point is the recognition that access to information is not equivalent

\footnotetext{
${ }^{1}$ Contribution presented at Quantum Interaction - 2014, Lihn in Filzbach, Switzerland, June 30th to July 4th, 2014. To be published in Springer Lectures Notes in Computer Science.
} 
to transparency. Instead transparency is intimately related to learning. In this paper we suggest that a quantum approach to information processing by human receptors may shed some fruitful light on the public debate as well as provide insights of value for practical applications.

The information system community, among others, was early aware that in addition to the physical constraints, the transmission of information e.g., about a new information system, is conditioned by cognitive limitations. Appealing to cognitive scientists they formulate those limitations in terms of "mental models", "cognitive frames" or "scripts" that simultaneously enable and constrain understanding. Gioia writes about "definitions of (organizational) reality that serve as vehicule for understanding and action" (Gioia [9], p.50). "They include assumptions, knowledge and expectations expressed symbolically through language, visual images, metaphors and stories. Frames are flexible in content and structure having variable dimensions that skift in salience and content by context and over time" (Orlikowsky and Gash [19]).

In economics, we talk about "framing effects" when alternative descriptions of one and the same decision problem induce different decisions from agents. Kahneman and Tversky [13] address framing effects by making the crucial point that "the true objects of evaluation are neither objects in the real world nor verbal descriptions of those objects; they are mental representations". To capture this feature, framing effects have been modelled as the "process of constructing a representation" as a measurement performed on the (quantum) state of the agent (LambertMogiliansky, Zamir and Zwirn [16]). In Lambert-Mogiliansky and Busemeyer [14], we show how this approach is consistent with a number of central theories in psychology including e.g., Self perception and provides a tractable approach to questions related to identity and self-control. In this paper, we introduce element of a theory of learning based on those premises and suggest that it provides a fruitful way to capture some of the above mentioned concerns expressed by practitioners.

We propose that the cognitive process involved in information processing includes two steps: i. receiving information framed according to some representation; ii processing information in terms of ones preferred representation. This learning process is not Bayesian as soon as we allow for Bohr complementarity of representations. The issue of learning by non-classically minded agents has been approached in Danilov and Lambert Mogiliansky $[4,5]$ and more recently in Busemeyer and Bruza [1].

A central result in learning theory is that Bayesian updating (under weak conditions) converges to the truth. However critics have been raised on several aspects of Bayesianism (see e.g. Gilboa, Postlewaite and Schmeidler [11]. Departures from Bayesian updating may occur because they undervalue or overvalue priors (e.g., Epstein Noor Sandroni [8]). Alternative approaches have been developed in connection with Maxmin Expected Utility (Gilboa and Schmeidler [10]) and Multriple priors (Hanany and Klibanoff[12]). Our approach implies a novel departure from Bayesian updating appealing to the recent success of the quantum formalism in psychology and in social sciences (for a survey of recent advances see Khrenikov [15] and Busemeyer and Bruza [1].

\section{1) Learning by non-classically minded agents}

It is a common place that human beings are not capable of holding very complex picture in mind (cf "small worlds" in Savage 1954). They consider reality focusing on one perspective at a time and show difficulty switching perspective as amply documented. What the quantum 
Transparency in Public Life - A Quantum Cognition Perspective

approach entails is that this inability to seize reality in its full richness has far reaching consequences beyond incomplete knowledge. When the perspectives are incompatible in the agent's mind, his understanding of reality does not look like a puzzle that is assembled progressively, instead the evolution of the picture of reality is subjected to disturbances (discrete jumps) which entails specific properties of the learning process.

In the present context the parallel with QM can be described as follows: the system is the subjective picture about the world, it is a psychic object. To gain information on the mental picture of the world two things can be done. One may add some information about the outside world. And one may perform a measurement of the picture by means of introspection. New information about the world transforms the picture (in a way similar to a preparation procedure). Thereafter, the agent processes information i.e., he "updates" his beliefs. This is modeled as a measurement that projects the new state (picture) onto one of the possible pictures defined by his preferred representation (see below).

Figure 1 is a well-known ambiguous picture. The mind may perceive a rabbit or a a duck. Both pictures are complete representations of the drawing: they confer a "meaning" to every detail. The brain oscillates between the two or settle for either one of them but cannot perceive both simultaneously. Both pictures are true but they are incompatible in the mind.

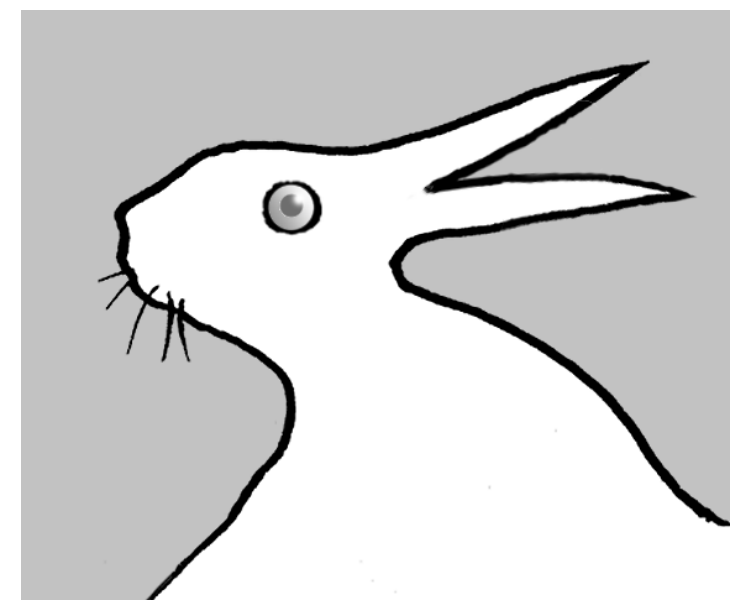

Figure 1. What do you see?

\section{1) The basic model}

Let the state (of understanding) of an agent be called a mental picture, we denote it $|\varphi\rangle \in \mathbb{H}$ where $\mathbb{H}$ is the Hilbert space of mental pictures of the (relevant) world. The present analysis addresses uncertainty exclusively linked to quantum indeterminacy reflecting cognitive constraints i.e., we shall be working exclusively with pure states. A distinctive feature of non-classical systems is the existence of dispersed pure states referred to as "intrinsic uncertainty". It is closely related to two other features of non-classical systems: the existence of incompatible measurements and the impact of the measurements on the state. ${ }^{2}$

\footnotetext{
${ }^{2}$ If a state is dispersion-free, i.e., the outcome of every possible measurement is uniquely determined, there is no reason for the state to change. If all pure states are dispersion-free then measurements do not impact on pure states and therefore all measurements are compatible. On the contrary, if a state is dispersed then by necessity it will be modified by an appropriate measurement. On the other hand, the change in a pure state is the reason for
} 


\section{- The notion of measurement}

Before entering into the analysis let us remind of some basic notions and how they relate to our issue. Generally, a measurement is an interaction between a system and some measurement device, which yields some result, the outcome of the measurement that we can observe and record. Two measurements are compatible if they, roughly speaking, can be performed simultaneously or more precisely, if the performance of one measurement does not affect the result of the other.

In our context a measurement is an introspective operation that acts on the agent's mental picture. The agent's asks himself a question and an answer, the outcome of the mental process is brought to consciousness. We propose that the cognitive limitations documented by practitioners can be modelled as the result of incompatible mental operations that act on the mental picture.

\section{- A preferred frame.}

We shall assume that the agent seeks information because he has some concern in mind e.g., he wants to make a decision. This assumption is consistent with psychological and neurobiological evidence that human cognitive processes (including perception) are structured by some form of intentionality.

Generally, a representation is an observable $R$ with eigenvalues $\left\{r_{1}, \ldots r_{n}\right\}$ interpreted as the possible "eigenpictures" belonging the representation $R^{3}$. The agent's state, a mental picture can written as a superposition of the possible eigenpictures in any representations of the (relevant) world:

$$
|\varphi\rangle=\sum_{i} \lambda_{i}\left|r_{i}\right\rangle, \quad \sum_{j} \lambda_{i}^{2}=1, \lambda_{i} \in \mathbb{R}
$$

A decision problem is defined as a correspondance from $D: \mathbb{H} \rightarrow A$ where $\mathbb{H}$ is the space of mental pictures of the world and $A$ the set of actions. For each (mentally represented) state of the world, it defines which actions the agent wants to undertake.

\section{Hypothesis 1}

There exists a representation $R^{*}$ such that $D$ is a coarsening of $R^{*}{ }^{4}$

Hypothesis 1 implies that if the agent receives (maximal) information framed in representation $R^{*}$ he will know exactly what to do. We also say that $R^{*}$ is fully congruent with $D$. Generally, $D$ has a lower dimensionality than $\mathbb{H}$, i.e., distinct eigenpictures can induce the same action.

\section{- The cognitive process.}

In our context, it is in place to explicitly decompose the cognitive process of learning into two steps:

\section{Step 1: Preparation}

The first step of the mental process is gaining information expressed within some representation $R \neq R^{*}$ (or frame - we use the terms interchangeably). It corresponds to a fully deterministic evolution of the mental picture. It operates on the initial state(picture) so as to project it onto

incompatibility of measurements. The initial outcome of a first measurement is not repeated because the system has been modified by a second measurement (see Danilov and Lambert-Mogiliansky $[4,5]$ ).

${ }^{3}$ All the representations $R, R^{*}$, etc. that we consider in this contribution have eigenspaces of dimension exactly equal to one. All these eigenstates are maximal information states for the individual.

${ }^{4} \mathrm{~A}$ measurement $M^{\prime}$ is coarser than $M$ if every eigenset of $M$ is contained in some eigenset of $M^{\prime}$, see Danilov and Lambert-Mogiliansky [4, 5] p. 334 . 
Transparency in Public life - A Quantum Cognition Perspective

the one eigenpicture reflecting the information content provided (see below for a concrete example). In the terminology of QM it resembles a process of preparation ${ }^{5}:|\varphi\rangle \rightarrow\left|\varphi^{\prime}\right\rangle=\left|r_{i}\right\rangle$, where $\left|r_{i}\right\rangle$ is an eigenpicture of the $R$ representation.

\section{Step 2: Measurement}

After having received the information, the agent processes information i.e., updates his mental picture $\left(r_{i}\right)$ with respect to his preferred representation $R^{*}$. This is a non-deterministic evolution which we model as a measurement. We first express $\left|\varphi^{\prime}\right\rangle=\left|r_{i}\right\rangle$ in terms of the eigenpictures of the preferred representation, $R^{*},\left|r_{i}\right\rangle=\sum_{j} \gamma_{j}\left|r_{j}^{*}\right\rangle$ applying $R^{*}$ we obtain

$$
R^{*}\left|\varphi^{\prime}\right\rangle \rightarrow\left|r_{j}^{*}\right\rangle \text { with probability } \gamma_{j}^{2}
$$

We see that after completing the cognitive process corresponding to interpreting the information in his own mental model, the agent's actual state of information is some $\left|r_{j}^{*}\right\rangle \neq\left|r_{i}\right\rangle$. The two states are not mutually exclusive (orthogonal) but they are incompatible in his mind which captures the cognitive limitation.

\section{2) Analysis: the value of new information}

We now develop a simple argument showing that information can increase the agent's relevant uncertainty. Uncertainty (intrinsic) is measured by the entropy of dispersion ${ }^{6}$ in terms of the preferred representation: let $|\varphi\rangle=\sum_{j} \nu_{j}\left|r_{j}^{*}\right\rangle$, since $\gamma_{j}^{2}$ is the probability for obtaining eigenpicture $\left|r_{j}^{*}\right\rangle$ after the measurement $R^{*}$, the (intrinsic) uncertainty associated with $\varphi$ is defined

$$
H(\varphi)=-\sum_{j} \nu_{j}^{2} \log \nu_{j}^{2}
$$

For the purpose of the argument, we shall assume that the agent's mental state is initially an eigenpicture of his preferred representation $|\varphi\rangle=\left|r_{2}^{*}\right\rangle$. This state reflects information that is perfectly congruent with $R^{*}$ but is not framed in $R^{*}{ }^{7}$. But the agent is not aware of $r_{2}^{*}$ unless he updates "his beliefs" which corresponds to the (measurement) operation of $R^{*}$. If he did, he would obtain

$$
R^{*}|\varphi\rangle=\left|r_{2}^{*}\right\rangle
$$

He would then be fully determined with respect to the decision problem. There would not be any (relevant) uncertainty left with $\nu_{2}=1$ we have $H(\varphi)=0$. But assume that instead he acquires new information relative to another but related aspects of the issue. We denote this representation $P$. This corresponds to steps 1 of the cognitive process above. The mental state $|\varphi\rangle$ is prepared into $\left|\varphi^{\prime}\right\rangle$ equal to some $\left|p_{i}\right\rangle$. "Enriched" with this new information, the agent now updates his mental picture with respect to his preferred representation (step 2):

$$
\left|\varphi^{\prime}\right\rangle=\left|p_{2}\right\rangle=\sum_{j} \gamma_{j}\left|r_{j}^{*}\right\rangle \rightarrow \text { any }\left|r_{j}^{*}\right\rangle \text { with probability } \gamma_{j}^{2} \neq 1
$$

We see that while he previously potentially "knew" $r_{2}^{*}$ (but was unaware of it) he is now is a state of hesitation where he believes that there is only a probability of $\gamma_{2}^{2}<1$ that the "true"

\footnotetext{
${ }^{5}$ In the process of preparation a system is put into a specific state.

${ }^{6}$ We talk about the entropy of dispersion rather than of probability distribution, because we are dealing with a pure state. See discussion above.

${ }^{7}$ In the example "learning that the administration lacks standard of ethics" is equivalent to learning "it is worthwhile to complaint". The point is before the agent processed the information in his own frame (step 2), he is not aware of this.
} 
eigenpicture is $r_{2}^{*} \cdot{ }^{8}$ The acquisition of new information has triggered the loss of some information leaving the agent in a state of intrinsic uncertainty captured by a strictly positive entropy:

$$
H\left(\varphi^{\prime}\right)=-\sum_{j} \gamma_{j}^{2} \log \gamma_{j}^{2}>0
$$

Proposition 1. Assume that the agent's mental state is one eigenpicture of his preferred representation, acquiring additional information in terms of a representation that is not compatible with the preferred one leads to confusion measured by increased dispersion entropy.

This is the central result of this paper. When two representations are incompatible in the agent's mind. Additional information is not always beneficial to the agent. It is unambiguously detrimental when the starting point is an eigenpicture of the preferred representation as illustrated in the argument above: the new information induces a strict loss of information implying confusion with respect to the concerns of the agent. More generally i.e., starting from an arbitrary eigenpicture (with non zero dispersion entropy) as in the example below, new information may either decrease or increase dispersion entropy.

We illustrate the result in Proposition 1 in figure 2 where the dimensionality of a representation is 2. The broad line corresponds to the preparation stage and the thin lines to the measurement.

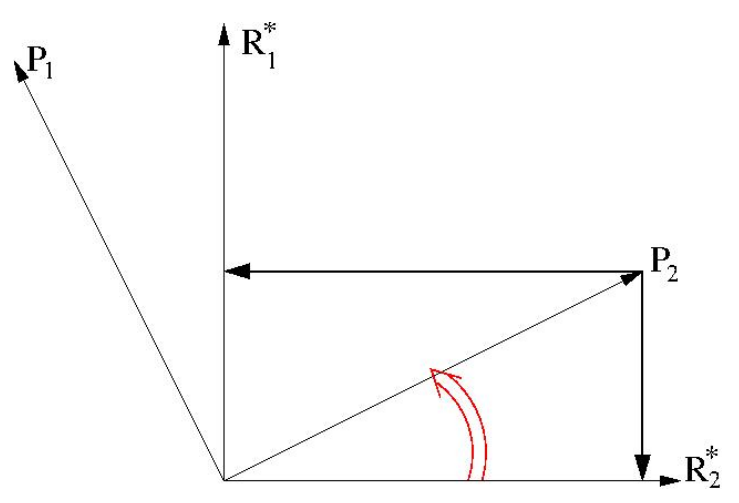

Figure 2. Detrimental information

Remark 1. In our case $r_{2}^{*}$ is true because it reflects an information congruent with $R^{*}$ and $\left|p_{i}\right\rangle$ is also true. The agent is not mistaken. He simply cannot hold in one single picture both informations. The introspective operation when processing information $p_{2}$ involves the whole (cognitively limited) mind and therefore upsets earlier held beliefs.

\section{- Classical cognition.}

In order to better understand the result above let us consider the classical counter-part of the argument above. It is well-known that the quantum model includes the classical one as a special case when all measurements commute. It is important for the argument that follows to understand that we are not considering all possible classical models but the classical model corresponding to the quantum one where all the premises are the same except that all measurements commute. We below discuss other possible classical approaches.

\footnotetext{
${ }^{8}$ After the introspective process, he will end up believing $r_{2}^{*}$ with some probability less than 1 .
} 
We next consider a classical situation where all representations are compatible in the citizen's mind. He can synthesize all information without constraint. Formally, this is expressed as follows. For simplicity let both $P$ and $R^{*}$ be two dimensional as in the illustrative example of figure 2. When $P$ and $R^{*}$ are compatible, a picture (a state) can have values in both representations simultaneously. This means that a state or picture can be represented as a vector in the four dimensional tensor product space $P \times R^{*}$ spanned by the eigenvectors $\left\{\left|p_{1} r_{1}^{*}\right\rangle,\left|p_{1} r_{2}^{*}\right\rangle,\left|p_{2} r_{1}^{*}\right\rangle,\left|p_{2} r_{2}^{*}\right\rangle\right\}$. Generally the picture (state of mind) $\psi$ of the citizen is as before expressed as a superposition of the basis vectors:

$$
|\psi\rangle=c_{1}\left|p_{1} r_{1}^{*}\right\rangle+c_{2}\left|p_{1} r_{2}^{*}\right\rangle+c_{3}\left|p_{2} r_{1}^{*}\right\rangle+c_{4}\left|p_{2} r_{2}^{*}\right\rangle, \quad \sum_{i=1}^{4} c_{i}^{2}=1 .
$$

Since we are dealing with a classical agent this can be interpreted as a belief state $b$ (a random variable) in boolean space $\Omega=\left\{\omega_{1}, \ldots, \omega_{4}\right\}$ with $\omega_{1}=p_{1} r_{1}^{*}, \omega_{2}=p_{1} r_{2}^{*}, \omega_{3}=p_{2} r_{1}^{*}, \omega_{4}=p_{2} r_{2}^{*}$ :

$$
b=\alpha_{1} \omega_{1}+\alpha_{2} \omega_{2}+\alpha_{3} \omega_{3}+\alpha_{4} \omega_{4}
$$

with $\alpha_{i}=c_{i}^{2}, i=1, \ldots, 4$ and reads as follows: the agent believes that with probability $\alpha_{1}$ the true state is $\omega_{1}$. Now in order to compare with the quantum case above assume that the agent initially has an information that is completely correlated with $r_{2}^{*}$ so $\operatorname{prob}\left\{r^{*}=r_{1}^{*}\right\}=0$ implying $\operatorname{prob}\left\{\omega=\omega_{1}\right\}=\operatorname{prob}\left\{\omega=\omega_{3}\right\}=0$. The priors $b_{0}$ writes

$$
b_{0}=\frac{\alpha_{2}}{\alpha_{2}+\alpha_{4}} \omega_{2}+\frac{\alpha_{4}}{\alpha_{2}+\alpha_{4}} \omega_{4}
$$

The agent now receives information in a way similar to the subsection above. So he receives information along $P$, he learns $p_{2}$ implying $\operatorname{prob}\left\{p=p_{1}\right\}=0$ implying $\operatorname{prob}\left\{\omega=\omega_{2}\right\}=0$. the agent processes that information i.e., updates his priors into posteriors

$$
b_{1}=\omega_{4} .
$$

The agent is now fully informed of the state of the world. So compared with the priors (2) there is no loss of information as in the quantum updating case.

Proposition 2. In the classical counter-part of the quantum model, additional information never increases the agent's uncertainty.

Proposition 2 underlines the implications of quantum cognitive limitations. In the absence of such limitations, i.e., when all representations are compatible in the mind of the agent, information is always beneficial and - in the classical counter part model - it reduces entropy. While if the citizen is cognitively limited, information can turn confusing.

Remark 2. It must be emphasized that the reasoning above relies on our assumption that we are dealing with a classical counter part where the agent learns that $r^{*}=r_{2}^{*}$ with probability 1 (corresponding to the pure state in the quantum model). If we relax slightly this assumption (i.e., allowing for incomplete information), the result in proposition 1 could be approached with a classical model of a different flavor. Assume the agent has priors that puts nearly all the weight (but not all) on $r_{2}^{*}$ so $\alpha^{1} \neq 0$ and $\alpha^{3} \neq 0$ as in $b$ in 1 . Next, he receives information $p^{2}$. If according to the agent's structural model, that information is highly correlated with $r_{1}^{*}$ (which is not the case in the original model) he updates his beliefs and puts more weight on $\omega_{1}$ and $\omega_{3}$ than he initially had. He is now less certain about what to do than he was before he got the information. His new beliefs exhibit a distribution with higher entropy. However, he has not lost 
information as in the quantum case. Instead the interpretation is that his priors were wrong so new information brought him closer to the truth. So a legitimate question is: are we only talking about interpretation or do quantum cognitive limitations really have a more profound bearing on learning?

\section{- Oscillating for ever.}

Bayesian learning operates within a Boolean algebra. The objective is to learn the parameters of the model of the system. ${ }^{9}$ Provided the priors are not inconsistent with the true model, we know (Schwartz 1965) that starting from any such priors Bayesian updating converges to the true state.

With quantum learning, we are in a Hilbert space, that is there exists a variety of resolutions of the system i.e., a variety of representations, of valid theories of the system. Those descriptions are not alternative to each other but complementary (in the sense of Bohr). As a consequence there exists no single true complete information state but a multiplicity of equally true maximal information states. Therefore, the state of knowledge of a quantum minded individual does not converge with new information but oscillates for ever. Consider again the example above. After the agent performed the introspective measurement $R^{*}$, if the agent is being asked about his understanding in $P$, he will perform a new measurement of the eigenpicture resulting from the first measurement and $p_{2}$ will not be recovered with probability 1 . Performing those measurements alternately, he will keep oscillating without converging i.e., without being able to settle for a definite value in both $P$ and $R^{*}$ simultaneously. ${ }^{10}$

Of course this example is simplistic and the agent might simply remember $p_{2}$. But in more sophisticated context, we expect the modification of the mental picture to be effective. Moreover, our focus is on the consequences for decision-making of lost information which are far from selfevident.

\section{2) Application: transparency in public life}

We next address the issue of providing access to public information and its relationship with transparency in terms of the theory developed above. Consider a situation where a citizen suspects that there has been some serious wrongdoing and he must decide whether or not to file a formal complaint against a public administration. Since that is costly to him he wants to collect information to evaluate his chance of winning.

The government makes available information e.g., on a website. Information can be expressed and organized according various principles. For instance some information concerns the extent of responsibility and discretion at various step of decision-making. Another informs about the ethical rules various bureaus obey. Yet, another set of information takes the perspective of the personnel and informs about the conditions for recruitment and promotion. The assumption is that the different perspectives are incompatible in the citizen's mind. When he thinks in moral/ethical terms, he cannot simultaneously envision the implication of the allocation of responsibilities for the quality of decisions or the quality competence emerging from

\footnotetext{
${ }^{9}$ Some consider also Bayesain updating with multiple priors (see e.g., Hanany and Klibanov [12]). But there is no consensus as to how to proceed - in sharp contrast with Bayesian updating of single priors.

${ }^{10}$ The general result is a transposition into cognition of the basic feature of quantum mechanics namely that it is not possible for complementary properties to have a determinate value simultaneously.
} 
the recruitment/promotion system. Therefore we define three non-commuting operators which for simplicity are two-valued: $R_{1}$ \{rule-bounded $(|R\rangle)$, discretionary $\left.(|D\rangle)\right\} ; R_{2}$ \{High ethical standards $(|E\rangle)$, No ethical standards $(|U\rangle)\} ; R_{3}$ \{Competent $(|C\rangle)$, Incompetent $\left.(|I\rangle)\right\}$. We assume one representation $R_{2}$ is fully congruent with $D$ the decision problem i.e., whether he should file a complaint of not. ${ }^{11}$ The initial state can be expressed in any of the representation:

$$
|\varphi\rangle=\lambda_{11}|R\rangle+\lambda_{12}|D\rangle=\lambda_{21}|E\rangle+\lambda_{22}|U\rangle=\lambda_{31}|C\rangle+\lambda_{32}|I\rangle .
$$

In figure 3 we depict the case when the citizen learns the administration is competent (green plain arrow) and when he learns the process is discretionary (blue plain arrow).

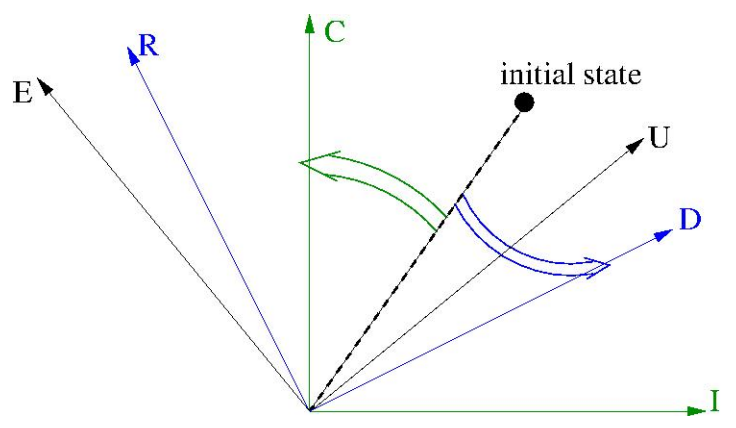

Figure 3. Transparency in public life; incompatible mental frames of an administration.

Recall that $R_{2}^{*}$ (in red) is fully congruent with the requirements of decision-making. When provided the information framed according to $R_{1}$ and learning e.g., that the administration is competent. Instead of decreasing uncertainty with respect to decision-making, the new information actually increases uncertainty (dispersion entropy) thereby creating confusion in the agent's mind. The initial state (doted line) was pretty close to $U$, the new state $C$ is almost equidistant to both $E$ and $U$. In contrast learning that the decision processes are discretionary (projecting onto $D$ ) though not fully congruent, reduces decision uncertainty. As we see the extent of congruence it is of paramount importance for cognitively constrained agents. In contrast with the classical model where at worst orthogonal information is useless, we know from proposition 1 that information can be detrimental; the citizens get confused (increased dispersion entropy).

Proposition 3. The government can increase citizens' confusion by providing information along a representation that is little congruent with the citizens' concerns.

This result implies that in our framework, truthful information can be used to manipulate the citizens away from their concerns in order to affect their decisions. The next section shows how full access to information can lead to confusion.

\section{- Sequential information acquisition: the architecture of the website.}

In this subsection we explore some implications of the results above for the architecture of the administration's website. Consider now a representation as a complete set of commuting observable. So for instance in $R_{3}$, beside competence (in terms of formal education), a description of the principles for selection in recruitment, of the principles governing evaluation for promotion,

\footnotetext{
${ }^{11}$ If the citizen pictures the administration as lacking ethical standard, that comforts his suspicion and he will definitely file a complaint. Conversely, if he is convinced the administration has high ethical standards, he will not file.
} 
a description of the rules and processes to be followed is provided (from the point of view of their complexity/simplicity), etc... Similarly for the other representations e.g., $R_{4}$ provides information about the standards for decisions, the appeal procedures etc...

Assume the website aims at providing full access to information. If the architect does not have in mind the quantum structure of the citizens' mind, the website will most probably be constructed so the progression in the tree mixes elements from different representations. Each new step entails a projection on a subspace of the representation to which the information belongs. But as we next show that has implication for the information previously "pictured", it is "lost" in the updating i.e., in the necessity for the citizen to be coherent i.e., fit information in one coherent to him picture.

To see this consider the following path. To facilitate the reading we let the eigenvalues of $R_{1}$ be labeled as $a_{i}$ and $R_{2}$ as $b_{i}$ the two representations are complementary in the mind of the citizen. Assume the citizen state is

$$
|\psi\rangle=\left|b_{1}\right\rangle=\sum_{i=1}^{3} \lambda_{i}\left|a_{1 i}\right\rangle
$$

This means that his knowledge is an eigenpicture of $R_{2}$, he has a determinate understanding of the administration in that representation. Now the website guides him further with information "non $a_{3}$ ". The picture of the citizen evolves from

$$
\left|b_{1}\right\rangle=\sum_{i=1}^{3} \lambda_{i}\left|a_{i}\right\rangle \rightarrow\left|\psi^{\prime}\right\rangle=\frac{\lambda_{1}}{\sqrt{\lambda_{1}^{2}+\lambda_{2}^{2}}}\left|a_{1}\right\rangle+\frac{\lambda_{2}}{\sqrt{\lambda_{1}^{2}+\lambda_{2}^{2}}}\left|a_{2}\right\rangle \neq\left|b_{1}\right\rangle
$$

So the citizen is less indeterminate with respect to $R_{1}$ but he lost the determination he had in $R_{2}$. With $\left|a_{i}\right\rangle=\sum_{j=1}^{3} \gamma_{i j}\left|a_{j}\right\rangle$ we can now write the citizen's state of information in $R_{2}$ :

$$
\left|\psi^{\prime}\right\rangle=\frac{\lambda_{1}}{\sqrt{\lambda_{1}^{2}+\lambda_{2}^{2}}} \sum_{j=1}^{3} \gamma_{1 j}\left|b_{j}\right\rangle+\frac{\lambda_{2}}{\sqrt{\lambda_{1}^{2}+\lambda_{2}^{2}}} \sum_{j=1}^{3} \gamma_{2 j}\left|b_{j}\right\rangle
$$

After redistribution in terms of the $b_{j}$ :

$$
\begin{aligned}
\left|\psi^{\prime}\right\rangle= & \left(\frac{\lambda_{1}}{\sqrt{\lambda_{1}^{2}+\lambda_{2}^{2}}} \gamma_{11}+\frac{\lambda_{2}}{\sqrt{\lambda_{1}^{2}+\lambda_{2}^{2}}} \gamma_{21}\right)\left|b_{1}\right\rangle+\left(\frac{\lambda_{1}}{\sqrt{\lambda_{1}^{2}+\lambda_{2}^{2}}} \gamma_{12}+\frac{\lambda_{2}}{\sqrt{\lambda_{1}^{2}+\lambda_{2}^{2}}} \gamma_{22}\right)\left|b_{2}\right\rangle \\
& +\left(\frac{\lambda_{1}}{\sqrt{\lambda_{1}^{2}+\lambda_{2}^{2}}} \gamma_{13}+\frac{\lambda_{2}}{\sqrt{\lambda_{1}^{2}+\lambda_{2}^{2}}} \gamma_{23}\right)\left|b_{3}\right\rangle .
\end{aligned}
$$

So we see that in $R_{2}$ the citizen is represented by a relatively complex superposition of all 3 possible eigenpictures of $R_{2}$. If he walks out from the website now, he is definitely more confused than when he started: he has no clear picture of anything. Adding yet another information belonging to a third representation will make his updating toward $R_{2}$ even more difficult.

Any learning process about a sufficiently complex system proceeds by step which can be identified as the sequential acquisition of coarse information. For quantum cognitively constrained citizens this corresponds to a sequence of preparation followed by possibly incompatible measurements. The more complex the system the more likely the cognitive constraints are binding i.e., the agent is not capable of synthesizing all information in one picture. Instead information is organized in a number of alternative perspectives of the system corresponding to alternative (incompatible) representations. We have the following claim

Claim i. Sequential acquisition of information can be particularly confusing if it mixes inputs from different representations. ii. Full access to information about a complex system is generally not beneficial to cognitively constrained agents. 
Transparency in Public life - A Quantum Cognition Perspective

The first claim is illustrated in the example above. The second claim follows from recognizing that the more complex a system, the more likely information about it involves a number of incompatible representations. The claim demonstrates the distinction between access to information and transparency. One implication is that an ill-intentioned webarchitect while providing full access to information can manipulate citizens so they give up their ambition to file a complaint whatever the initial state of mind. In other words transparency can be defeated precisely by providing full access to information.

\section{Concluding remarks}

This paper is a first exploration of learning by cognitively limited agents where the limitations are modeled appealing to quantum like characteristics of the mind. A main motivation is that the quantum approach has shown successful in explaining behavioral anomalies in decision-making while it also seems able to capture concerns expressed by practitioners dealing with information transmission and communication. In particular, the fact that i. people reason about reality within the frame of some a representation ("mental script") or as we call it, a mental picture, ii, the multiplicity of potential representations of reality generates specific problems for information transmission and learning. We model cognitive limitations in terms of the multiplicity of Bohr complementary mental representations of one and the same reality. We find that under intrinsic uncertainty, additional information may contribute to increasing the confusion of people. The analysis reveals a new significance of informational congruence which allows shedding some light on the issue of transparency in public life. In particular, we find that indeed access to information is not equivalent to transparency and that even truthful and full access to information can be exploited to manipulate cognitively constrained people. Future research will aim at extending the argument to a setting with uncertainty due to both incomplete information and intrinsic uncertainty. It will also establish the asymptotic properties of learning by quantum minded agents and perform a systematic comparison with non-Bayesian learning models.

\section{References}

[1] Busemeyer J.R. and Bruza P. (2012), Quantum Models of Cognition and Decision, Cambridge University Press.

[2] Busemeyer J.R., Wang, Z. and Townsend J.T. (2006), "Quantum Dynamics of Human Decision-Making", Journal of Mathematical Psychology, 50, p. 220-241.

[3] Busemeyer, J. R., Weg, E., Barkan, R., Li, X., \& Ma, Z. (2000), "Dynamic and consequential consistency of choices between paths of decision trees", Journal of Experimental Psychology: General, 129, p. 530-545.

[4] Danilov V. I. and A. Lambert-Mogiliansky (2008), "Measurable Systems and Behavioral Sciences". Mathematical Social Sciences 55, p. 315-340.

[5] Danilov V. I. and A. Lambert-Mogiliansky (2008), "Decision-making under non-classical uncertainty", in Proceedings of the the second interaction symposium (QI 2008), p. 83-87.

[6] Danilov V. I. and A. Lambert-Mogiliansky (2010), "Expected Utility under Non-classical Uncertainty", Theory and Decision 2010/68, p. 25-47. 
[7] Dawes S., (2010) "Stewardship and Usefulness: Policy principles for Information-based Transparency", Government Information Quartely, 27/4, p. 377-383.

[8] Epstein L, J. Noor and A. Sandroni (2008), "Non-Bayesian updating: A theoretical framework", Theoretical Economics 3, p. 193-229.

[9] Gioia D. (1986) "Symbols, Script and Sense-making: creating meaning in the organizational experience", in Thinking Organization H. Sims and Jr D. Gioia and Associates (eds) Jossey Bass San-Fransisco 1986, p. 49-74.

[10] Gilboa I. and D. Schmeidler (1989), "Maxmin Utility with non-unique priors", Journal of Mathematical Economics, p. 141-153.

[11] Gilboa I, A. Postlewaite and D. Schmeidler (2012), "Rationality of Beleifs or: why savage's axioms are neither necessary nore sufficient for rationality", Synthese, 187, p. 11-31.

[12] Hanany E. and P. Klibanov (2007), "Undating prefrences with multiple priors", Theoretical Economics, p. 261-298.

[13] Kahneman D. and A. Tversky (Eds.) (2000), Choices, values and frames, Cambridge University Press, New York.

[14] Lambert-Mogiliansky A., Busemeyer J.R. (2012), "Quantum indeterminacy in Dynamic decision-making: Self-control through identity management", Games, vol. 3/2, p. 97-118.

[15] Khrennikov A. (2010), Ubiquitous Quantum Structure - From Psychology to Finance, Springer.

[16] Lambert-Mogiliansky A., S. Zamir and H. Zwirn (2009), "Type indeterminacy - A Model of the KT(Khaneman Tversky)- man" Journal of Mathematical Psychology, vol 53/5, p. 349361.

[17] La Mura P. (2009), "Projective Expected Utility" Journal of Mathematical Psychology, p. $408-414$.

[18] Noveck B. (2009), "Wiki Government: How Technology Can Make Government Better, Democracy Stronger, and Citizens More Powerful", Brookings Institution Press, Washington DC.

[19] Orlikowsky W.J. and D.C. Gash (1994), "Technological frames: making sense of information technology in organizations", ACM Transactions on Information Systems (TOIS) - ACM Transaction on Information Systems, vol 12/2, p. 174-207.

[20] Savage L. (1954), The Foundation of Statistics, Dover publication inc, New York. 